\title{
CELL STRATIFICATION, SPHEROID FORMATION AND BIOSCAFFOLDS USED TO GROW CELLS IN THREE DIMENSIONAL CULTURES
}

\author{
Hana Hrebíková, Dana Čǐžková, Jana Chvátalová, Rishikaysh Pisal, Richard Adamčik, Pavel Beznoska, \\ Daniel Díaz-Garcia, Jaroslav Mokrý
}

\author{
Department of Histology and Embryology, Charles University Medical Faculty, Hradec Králové, Czech Republic
}

\begin{abstract}
Summary: The cell culture became an invaluable tool for studying cell behaviour, development, function, gene expression, toxicity of compounds and efficacy of novel drugs. Although most results were obtained from cell cultivation in two-dimensional (2D) systems, in which cells are grown in a monolayer, three-dimensional (3D) cultures are more promising as they correspond closely to the native arrangement of cells in living tissues. In our study, we focused on three types of 3D in vitro systems used for cultivation of one cell type. Cell morphology, their spatial distribution inside of resulting multicellular structures and changes in time were analysed with histological examination of samples harvested at different time periods. In multilayered cultures of WRL 68 hepatocytes grown on semipermeable membranes and non-passaged neurospheres generated by proliferation of neural progenitor cells, the cells were tightly apposed, showed features of cell differentiation but also cell death that was observable in short-term cultures. Biogenic scaffolds composed of extracellular matrix of the murine tibial anterior muscle were colonized with $\mathrm{C} 2 \mathrm{C} 12$ myoblasts in vitro. The recellularized scaffolds did not reach high cell densities comparable with the former systems but supported well cell anchorage and migration without any signs of cell regression.
\end{abstract}

Keywords: 3D cell culture; Hepatocytes; WRL 68 cells; Neurosphere; C2C12 cells; Bioscaffold

\section{Introduction}

Cell culture offers the consistency and reproducibility of results and for that reason it is used as a valuable tool in biological research. It became a common approach for the investigation of morphological, biochemical and physiological properties of cells as well as large scale manufacturing of biological compounds. Conditions that allow growing cells in vitro vary according to a type of the cells (5). They create the artificial environment in which the cells are maintained in a suitable dish containing a culture medium. Some cell types can be grown floating in suspension culture while the others require attachment to a solid or semisolid substrate. When cells with migratory and proliferative potential are cultured in vitro on an adhesive surface they grow in a sheath to form a monolayer. This anchorage-dependent approach to cell cultivation allowed studying biology of individual cells, to establish continuous cell lines, which laid down the basis for modern cellular and molecular biology. However, traditional two-dimensional (2D) systems have a number of drawbacks. Most importantly they do not reflect closely the conditions under which the cells interact in living tissues. Behaviour of cells grown in planar systems differs from those observed in vivo. The cells cannot maintain correct morphology and adopt flattened shapes (26).

Retention of histological structure and organotypic function is possible when the cells are grown in three-di- mensional (3D) culture systems $(4,13)$. Cells grown in 3D contexts closely resemble in vivo situation as they respond to the geometry of their environment and also display excellent functional characteristics. There are two major approaches to $3 \mathrm{D}$ cultivation (6). The first is based on explantation of whole fragments of tissue that are maintained in an organ culture. The second recombines the cell lineages using histotypic or organotypic cultures. 3D systems are suitable for the developmental studies, screening and drug discovery procedures, modelling disorders including malignant invasion in vitro and testing new therapies. Last but not least, the need for $3 \mathrm{D}$ culture systems is crucial for tissue engineering and preparation of new tissue constructs.

In this study we describe and compare morphological analyses of three different approaches for generation of histotypic cultures: multilayered cultures of WRL 68 hepatocytes grown on semipermeable membranes, spheroids generated by neural stem/progenitor cells called neurospheres, and acellular muscle scaffolds recellularized with C2C12 myoblasts.

\section{Material and Methods \\ Cultivation of WRL 68 cells}

WRL 68 cell line (ATCC, USA) of human fetal hepatocytes was cultured in EMEM medium (BioWhittaker, Cambrex, 
USA) supplemented with $10 \%$ fetal bovine serum (FBS; Gibco, Invitrogen USA), glutamine, penicillin $100 \mathrm{U} / \mathrm{ml}$ and streptomycin $100 \mu \mathrm{g} / \mathrm{ml}$ (Sigma-Aldrich, Czech Republic) at $37{ }^{\circ} \mathrm{C}$ in a humid atmosphere of $95 \%$ air and $5 \%$ carbon dioxide. WRL 68 cells were grown in a monolayer or $3 \mathrm{D}$ cultures. A monolayer was obtained by growing the cells in culture flasks (Nunc, Denmark) and passaging them twice per week (split ratio $1: 15$ ). Cells in a monolayer were grown for 9 days only. For a long-term (12 weeks) and 3D growth in vitro, 250,000 cells were cultured on a special $25-\mu \mathrm{m}$ thick plastic bottom membrane in PetriPerm dishes (Vivascience, Germany) that is well permeable to oxygen and carbon dioxide. The culture medium was changed three times a week.

The viability test was performed with propidium iodide $50 \mu \mathrm{g} / \mathrm{ml}$ added to the culture for $10 \mathrm{~min}$ after a thorough washing of cells with PBS under TE 300 Eclipse (Nikon, Japan) equipped with epifluorescence.

\section{Neurosphere assay}

Suspension of cells isolated from the developing rat brain on embryonic day 14 was obtained after trypsinization and gentle mechanical trituration using a micropipette in glucose-saline solution containing DNase I. The neural cells were plated at a density 15,000 cells/ $\mu \mathrm{l}$ in a chemically-defined serum-free medium composed of a 1:1 mixture of DMEM (Gibco) and Ham's F12 nutrient containing N2 (Gibco) and B27 supplement (Life Technologies, MA, USA) with $10 \mathrm{ng} / \mathrm{ml} \mathrm{bFGF}$ and $20 \mathrm{ng} / \mathrm{ml}$ EGF (PeproTech, UK). Cultures were maintained in medium at $37^{\circ} \mathrm{C}$ in humidified atmosphere consisting of $95 \%$ air and 5\% carbon dioxide. Half the volume of the medium was replaced twice a week with a fresh medium.

\section{Cultivation of C2C12 cells}

C2C12 mouse adherent myoblasts (Sigma-Aldrich) were grown in Dulbecco's modified Eagle's medium (DMEM) supplemented with heat-inactivated $10 \%$ fetal bovine serum, $2 \mathrm{mM}$ glutamine, penicillin $100 \mathrm{U} / \mathrm{ml}$ and streptomycin $100 \mu \mathrm{g} / \mathrm{ml}$ (Sigma-Aldrich), $\mathrm{pH}$ 7.5. After reaching 80\% confluency after 2-3 days in vitro the cells were passaged. The cell line was maintained in a $5 \% \mathrm{CO}_{2}$ atmosphere at $37^{\circ} \mathrm{C}$ and cell viability was assessed by the Trypan Blue (Life Technologies, MA, USA) exclusion test. For scaffold recellularization, 2 to 3 acellular scaffolds were placed in 6 well plates dishes and these were exposed to $10^{5}$ cells per $\mathrm{ml}$ of cultivation medium. Cultivation medium was replaced with fresh solution every three days.

\section{Preparation of decellularized muscle scaffolds}

All procedures were approved by the Ethical Committee supervising procedures on experimental animals at Charles University Medical Faculty in Hradec Králové. Tibialis an- terior (TA) muscle was isolated from sacrificed C57Bl/6J mice and cut in pieces $3 \times 6 \mathrm{~mm}$. After washing with PBS buffer, muscle pieces were incubated in sterile $5 \mathrm{M} \mathrm{NaCl}$ in distilled water for $2 \mathrm{~h}$ at $4{ }^{\circ} \mathrm{C}$ under slow agitation and then immersed into hypotonic $10 \mathrm{mM}$ TrisHCl for $22 \mathrm{~h}$ at $4{ }^{\circ} \mathrm{C}$ under slow agitation. The next step included incubation with $1 \%$ SDS in distilled water for $24 \mathrm{~h}$ at room temperature under slow agitation. Scaffold was sterilized by $0.1 \%$ peracetic acid solution titrated to $\mathrm{pH} 7.0$ for $4 \mathrm{~h}$ at room temperature under slow agitation. PBS buffer applied for $24 \mathrm{~h}$ at $4{ }^{\circ} \mathrm{C}$ assured washing out cell residues. DNA content in scaffolds was decreased with DNase (Promega, WI, USA) applied for $1 \mathrm{~h}$ at $37^{\circ} \mathrm{C}$ followed by washing with fresh PBS buffer.

\section{Histology}

3D cell formations yielded from cultures were processed for histology to assess the cell morphology and ratio of cell differentiation. The samples were immersed in neutral buffered formalin for $30 \mathrm{~min}$ at room temperature. The scaffolds were fixed by $4 \%$ paraformaldehyde (Sigma-Aldrich) overnight. Following dehydration with increasing concentrations of ethanol and exposure to xylene, the samples were embedded in melted paraffin. After cooling the tissue blocks were cut to $7-\mu \mathrm{m}$ thick sections using sliding microtome Leica SM2000R. The sections were attached to the slide with alum gelatine and after deparaffinization and rehydration they were stained with hematoxylin and eosin, Masson's green trichrome or processed for immunocytochemistry.

Alternatively the samples were fixed in mixture of $1 \%$ paraformaldehyde and $1 \%$ glutaraldehyde (Sigma-Aldrich) for 4 hours and following a thorough washing with phosphate buffer they were fixed with $2 \%$ osmium tetroxide (SPI Supplies, PA, USA) for $90 \mathrm{~min}$ and immersed in $2 \%$ uranyl acetate (Sigma-Aldrich) for $30 \mathrm{~min}$. After washing with sodium acetate and $10 \%$ saccharose, the tissue was dehydrated with increasing concentrations of ethanol, transferred to propylene oxide (Sigma-Aldrich) and embedded in Durcupan-Epon resin (Sigma-Aldrich). The tissue blocks polymerized at $60{ }^{\circ} \mathrm{C}$ for 60 hours and then the semithin sections were cut on Ultrotome Nova (LKB, Sweden) and stained with toluidine blue.

\section{Immunocytochemistry}

Sections of WRL 68 cells were incubated for $20 \mathrm{~min}$ in methanol containing $1 \% \mathrm{H}_{2} \mathrm{O}_{2}$ to reduce endogenous peroxidase activity. For antigen retrieval, the sections were exposed to microwaves $(700 \mathrm{~W})$ in sodium citrate solution for $2 \times 5 \mathrm{~min}$. After thorough washing in $0.2 \mathrm{M}$ Tris- $\mathrm{HCl}$ buffer containing $0.5 \%$ Triton X-100 (Sigma-Aldrich), the sections were exposed to primary antibodies against M30 neoepitope (Roche Life Science, IN, USA) for $45 \mathrm{~min}$. After washing, sections were incubated with anti-mouse secondary biotinylated antibody (Sigma-Aldrich) for $45 \mathrm{~min}$ and then with streptavidin labelled with horseradish peroxidase 
(BioGenex, CA, USA). After rinsing, the reaction was developed using DAB (3,3'-diaminobenzidine tetrahydrochloride, Sigma-Aldrich) and $\mathrm{H}_{2} \mathrm{O}_{2}$. Sections were then counterstained with methyl green, dehydrated and mounted in DPX mounting medium (Sigma-Aldrich).

\section{Results}

\section{$3 D$ cultures of WRL 68 cells}

WRL 68 cells proliferated briskly and in 3-4 days in vitro they reached $70 \%$ confluence (Fig. 1A). Propidium iodide labelled only a minor fraction of cells $(0.95-1.48 \%)$. Without passaging the cells reached $100 \%$ confluence within a week and started to grow over each other. However, basal cells overgrown by new overlaying cell layers suffered with deficit in oxygen, changed their morphology and started to degenerate and ultimately die.

Cultivation in PetriPERM dishes avoided the initial cell degeneration due to cell hypoxia and allowed the cell stratification. In the second week the areas containing several cell layers were separated by areas covered by a cell monolayer. An intense growth is reflected by the finding of mitotic cells inside of the multilayered structure (Fig. 1B and $1 \mathrm{C}$ ). After 3 weeks in vitro, the whole bottom of the PetriPERM dishes was covered by several cell layers; the thickness of a cell layer varied from 2 to 8 cells. Histological examination revealed the basal cells assumed a flattened morphology, inner lying cells were polygonal whereas the upermost cells had spherical or oval shapes. Almost all the cells were in contacts with cell membranes of other WRL 68 cells. The cells contained one to two cell nuclei and pale but basophilic cytoplasm. Rarely cells undergoing cell death were encountered. The eosinophilic cytoplasm of apoptotic cells surrounded pycnotic nucleus; some nuclei were fragmented. After a complete decay of few adjacent cells small empty spaces were left between the viable cells; rarely apoptotic bodies were encountered inside of these spaces. After a 6-week cultivation the cells accumulated in 8-9 layers. However, the number of viable cells decreased and as a result only some cells were in a complete contact with their neighbours. The cells of a basal layer remained viable even in a long-term culture. Other viable cells were found as single cells not forming intercellular junctions. They had a large cell nucleus and pale basophilic cytoplasm. The number of cells with the eosinophilic cytoplasm increased. The cellular mass contained the cells reflecting different features of programmed cell death: condensed chromatin, karyorrhexis and apoptotic bodies. Eosinophilic cells expressed M30 neoepitope confirming that cells were dying by apoptosis (Fig. 1D). Intensity of an immunoreactive signal varied; some cells were stained weakly while the others (especially small cells and apoptotic bodies) expressed a strong signal in their cytoplasm. M30-positive cells were of different sizes and bizzare shapes whereas negative cells were oval or polyedric. Histological examination of sections through the
PetriPERM membrane covered with layers of WRL cells harvested from culture after 12 weeks identified eosinophilic cellular mass consisting of degenerating cells. There were only few viable cells often connected together. Nevertheless, a basal layer of flattened cells remained well preserved.
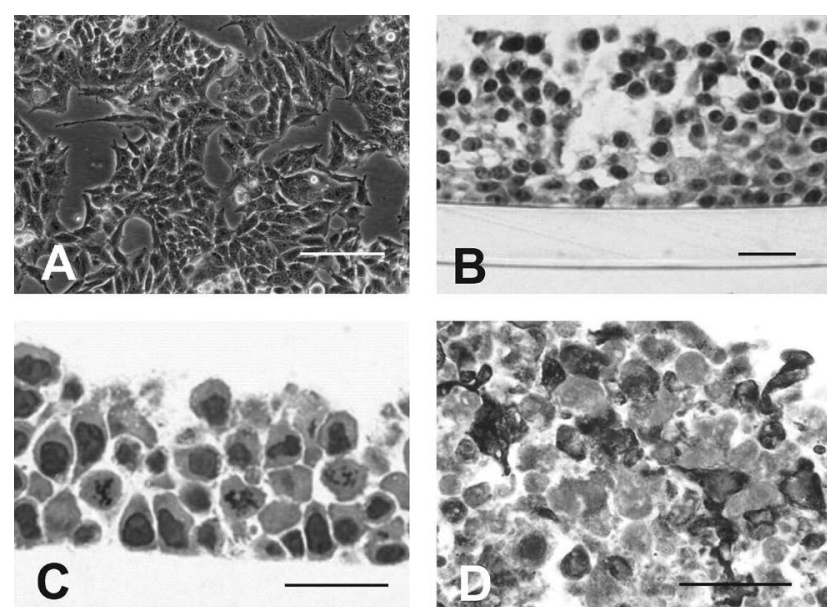

Fig. 1: Culture of WRL 68 cells. A) Phase contrast of a WRL 68 cell monolayer 3 days after seeding showed the subconfluent cells with a uniform morphology. Rounded cells corresponded to dividing and dying cells as both events involved retraction of cytoplasmic processes and detachment from junctions with adjacent cells. B) After a 9-day cultivation in a PetriPerm dish (visible at bottom) WRL 68 cells grew over the others in several layers. Small cavities and empty spaces appeared among the cells but the basal layer remained always continuous. C) A detailed view of a shortterm (9-day) culture with cells arranged in 5 layers. The cells were mononucleated, uniform in morphology and size and connected via intercellular junctions; few cells underwent mitotic division. D) Immunohistochemical detection of M30 neoepitope gave evidence of frequent apoptosis in cells harvested from a long-term (12-week) culture. The positive cells had bizzare shapes; the signal was absent from the cell nucleus, which often shrinked. The dying cells were localized between intact cells in all cell layers of a 3D culture. Scale bar $50 \mu \mathrm{m}$ in A, $25 \mu \mathrm{m}$ in B-D.

\section{$3 D$ cultures of neural progenitor cells}

In serum-free cultivation media differentiated neural cells rapidly died and only neural progenitor and stem cells survived and responded to stimulation with growth factors by repetitive cellular divisions. The newly generated cells remained adhered together, which resulted in formation of multicellular floating spheroids, called neurospheres. Under ideal conditions, each neurosphere represented a clone of a single founder cell, however, at high cell densities, some cells could adhere and small neurospheres were also fusing together forming larger spheroids within few days. The edge of neurospheres had sharp and smooth contours (Fig. 2A). Without passaging the neurospheres increased their sizes rapidly in the course of the first two weeks in vitro. At the end of the first week many neurospheres reached sizes over $50 \mu \mathrm{m}$. 
Histological examination revealed that densely packed small cells occupied the superficial 2-4 layers (Fig. 2B). The peripheral cells were basophilic and some underwent mitotic division. A cellular density in a central region of larger neurospheres was lower when compared with peripheral areas. Rarely fragments of pycnotic chromatin were also noticed demonstrating that cell death also occurred in early neurospheres. Semithin sections of neurospheres harvested in the second and third week of cultivation showed similar results. The cells started to differentiate and as a result the cell population was heterogeneous. Superficial cells were more immature than cells lying in the central region: they were smaller and densely arranged. Mitotic figures were not frequent but if they appeared they were localized to the layers of superficial cells. All nuclei of cells occupying the central core were in the interphase and they often contained the nucleolus. Bodies of these cells were larger and paler and sent elongated cytoplasmic processes. Apoptotic cells or apoptotic bodies observed between the groups of viable cells were more frequent than dividing cells (Fig. 3C). These cells were small, contained fragments of condensed chromatin and represented $3-5 \%$ of all cells. Small cavities that had sizes of cells were distributed over the neurosphere and sometimes

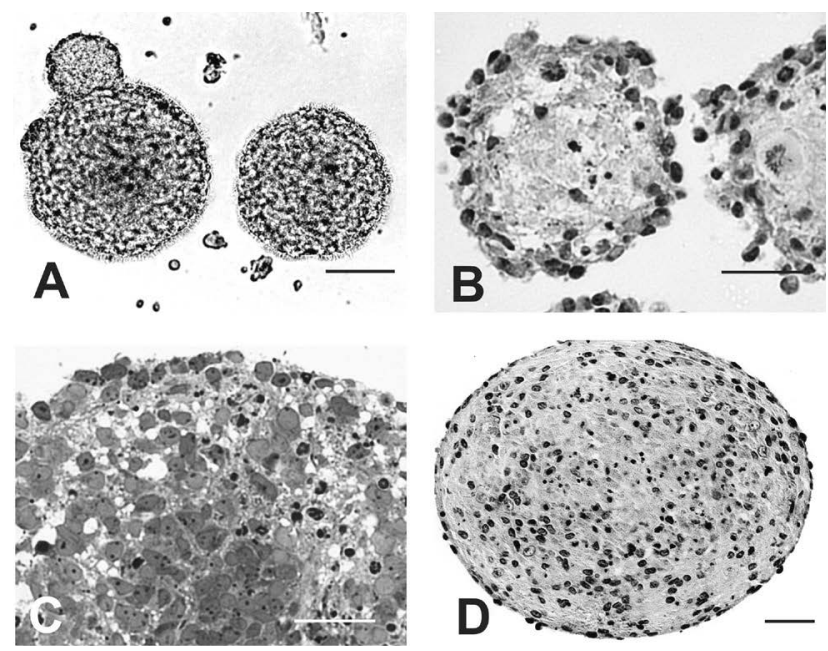

Fig. 2: Culture of neurospheres. A) Phase contrast of nerospheres after 5 days in vitro showed their regular shapes and outlines. The left neurosphere fused with smaller one. B) Semithin sections revealed the inner structure of the early neurospheres: The periphery was occupied by small basophilic cells arranged in few layers; mitotic divisions were confined to this zone. Central areas were less cellular and contained cells with pycnotic and fragmented chromatin. C) In 9-day neurospheres, programmed cell death was relatively common. Cells with pycnotic and fragmented nuclei were usually seen in areas with small empty spaces. Central areas accumulated lots of differentiating cells with cytoplasmic processes. Intact cells in the interphase overnumbered the dying cells. D) In long-term cultures, dying cells were distributed in central less cellular areas. A paraffin-embedded section of an 8-week neurosphere showed intact and differentiated cells in a peripheral belt. Scale bar $50 \mu \mathrm{m}$ in A, B, D, $25 \mu \mathrm{m}$ in C. contained a shrinked dying cell. After one and two months of cultivation in a dish the neurospheres were still viable, they grew larger and showed similar microscopic features. A central region contained cells that showed regular signs of cell regression with eosinophilic cytoplasm and shrinked chromatin (Fig. 2D). A peripheral region still contained viable and intact cells. Differentiated cells were localized to deeper layers whereas less mature cells occupied outermost layers. If the neurosphere attached to a bottom of a culture dish, the neurosphere basis contained less cells than the free edges.

\section{$3 D$ scaffolds colonized with C2C12 cells}

C2C12 cells plated in dishes first had typical starshaped or fusiform morphology. Only dividing cells were rounded. As mononucleated cells became closely adherent they elongated. After four days, first cells started to fuse. The cell fusion gave rise to multinucleated structures that grew larg-
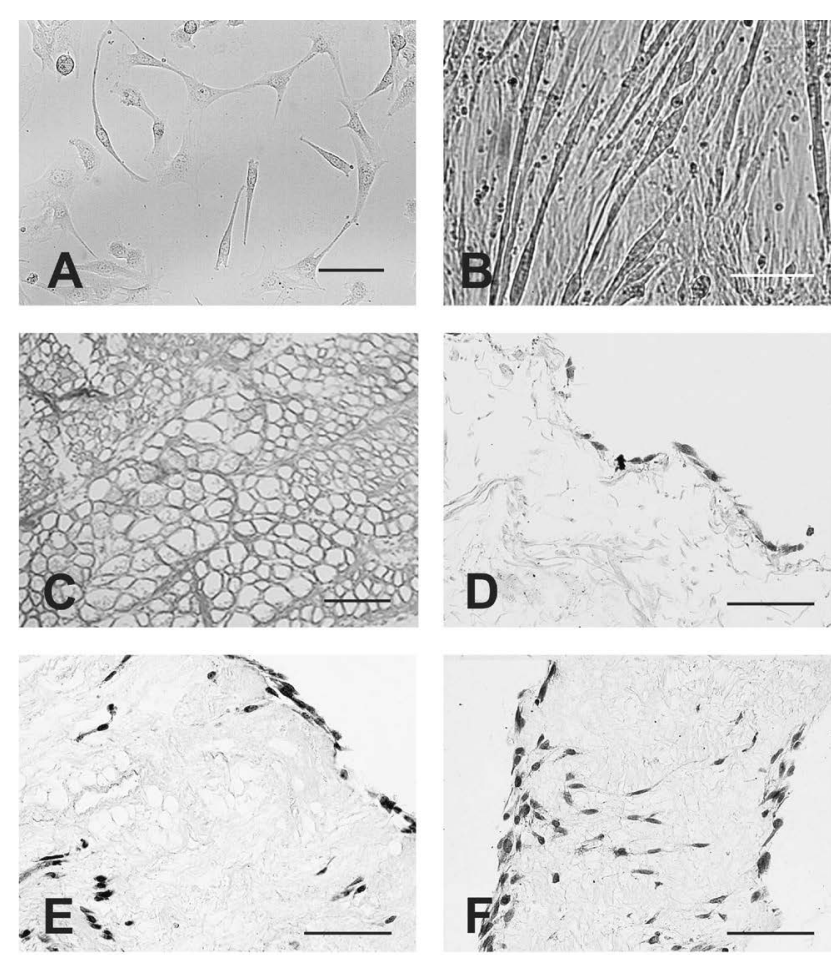

Fig. 3: Culture of $\mathrm{C} 2 \mathrm{C} 12$ cells and scaffolds. A) Phase contrast of $\mathrm{C} 2 \mathrm{C} 12$ cells 2 days from passaging showed a spindle shaped to flatten morphology of mononucleated myoblasts. B) Cultivation in $10 \%$ of horse serum resulted in cell fusion and appearance of elongated myotubes containing multiple nuclei. C) Histology of an acellular muscle scaffold confirmed absence of myofibres and cell nuclei. Extracellular matrix was well preserved and reflected a basic structural plan of the skeletal muscle. D-F) Scaffolds recellularized with C2C12 cells 3- (D), 6- (E), and 9-day (F) cultivation were covered with viable cells that started to penetrate inside. Most cells were mononucleated although a close cell apposition was observable on day 9. Scale bar $25 \mu \mathrm{m}$ in A, $50 \mu \mathrm{m} \mathrm{B}, 100$ in C and $150 \mu \mathrm{m}$ in D-E. 
er reaching the length of several hundred micrometers. In confluent cultures these myotubes appeared regularly. For colonization of acellular scaffolds undifferentiated mononucleated myoblasts were used. The scaffolds devoid of cells were cut to small pieces with the length not exceeding $3 \mathrm{~mm}$ and diameter below $2 \mathrm{~mm}$ before they were exposed to $\mathrm{C} 2 \mathrm{C} 12$ cells. Decellularized scaffolds were processed for histology as a control prior seeding with cells. Masson's Green trichrome staining gave evidence that extracellular matrix, namely collagen fibres and bundles, was well preserved and organized in a muscle tissue-specific manner. Empty spaces left after a removal of muscle fibre cytoplasm showed parallel arrangement and were delineated with the thin endomysium. The perimysium, epimysium and tendons contained more abundant extracellular matrix. Lumina of sporadic medium-sized blood vessels running through the tissue were observable but they lacked a simple squamous endothelial lining. Histological examination verified the absence of any cells and muscle fibres; fluorescence was used to confirm that no cell nuclei were left in decellularized scaffolds. Re-seeding of scaffolds with $\mathrm{C} 2 \mathrm{C} 12$ cells confirmed the murine myoblasts well adhered to its extracellular matrix and they remained viable. Cell morphology and cell migration along the scaffolds was examined in paraffin-embedded sections at 3, 6 and 9 days after cultivation. Three days in vitro the cells migrating over the outer surface adopted bipolar and flattened morphology. The cells had basophilic cytoplasm and extended thin cytoplasmic processes. They were mostly arranged in a single discontinuous layer; cells overlapping did not occur. Three days later, on day 6 of their in vitro cultivation, $\mathrm{C} 2 \mathrm{C} 12$ cells attaching to the outermost surfaces increased in cell numbers and formed one to three continuous layers. Some cells entered inside of the scaffolds and migrated through the extracellular matrix as single cells. The cells were elongated with basophilic cytoplasm and single nucleus. Inside the scaffold the cells formed small groups usually on top surfaces of collagen bundles. By day 9 in vitro more cells accumulated in some deep areas of scaffolds while the other places were left unoccupied which likely reflected a varying accessibility. The myogenic cells entering inside represented less than $10 \%$ of all cells that remained in a contact with the scaffold. Interior cells were arranged in the same orientation in space as they occupied tubular spaces left after removal of original skeletal muscle fibres. Few cells that came to a close contact started to fuse but the number of multipotent cells was still very small. Majority of cells that covered the outer surface or recellularized interior of scaffolds were of bipolar to fusiform shapes and mononucleated. No myotubes, polyploidy giant cells or dying cells were detectable in short-term cultures of recellularized scaffolds.

\section{Discussion}

Cells of multicellular organisms are in mutual contacts with the neighbouring cells and components of extracellular matrix that are arranged in all directions. The cells can be isolated from the tissues and grown in vitro to study their complex biological functions. Most current approaches prefer cell cultivation as a monolayer due to simplicity although it does not reflect the whole tissue complexity. Nevertheless 2D systems allowed optimization of basic culture conditions including composition of cultivation medium and its supplementation with growth factors.

$3 \mathrm{D}$ cell cultivation requires different approaches. There are few general requirements such as sufficient cell viability, growth, adherence, differentiation potential and stability. This study described data for 3D cultures prepared by stratification of cells grown on a semipermeable membrane, forming spheroids in a non-adherent system and supporting cell migration along the $\mathrm{z}$-axis with a suitable scaffold.

We utilized WRL 68 cells for cultivation of hepatic cells as the WRL 68 cells can be split in new culture dishes with the ratio $1: 20$ after passing. Adult liver cells exhibit low growth potential and cannot be maintained in culture for long time limiting their study to few days in vitro. A primary culture of embryonic hepatocytes can be grown for few weeks due to initially large proliferative capacity; however, they cannot be passaged successfully. A high mitotic activity of WRL 68 cells allows cell overlapping and stratification, which is a feature of complex epithelia. Diffusion of oxygen and nutrients is a limiting factor counterbalancing number of cell layers and thickness of the entire multicellular formation. With an increasing size the centrally lying cells are not well nourished and undergo regression. Apoptotic WRL 68 cells expressed M30 neoepitope formed by cleavage of cytokeratin 18 (Fig. 1D). Cultivation of cells attached to semipermeable dishes supplied the $3 \mathrm{D}$ cell formations with oxygen allowing them to reach larger sizes. But in the long-term culture frequent cells enter cell death. Although the WRL 68 cells grown in a long-term 3D culture increase expression of the membranous transporters Mdr1 and Mrp2 (3) they do not reflect pattern of polarized hepatocytes. One prerequisite for cell polarization is making contacts with variety of multiple cell types that participate in tissue construction to form its stroma, nerves and blood or lymphatic vessels. Co-culture of different cell types could yield better results. For example the hepatocyte-sinusoid alignment supports liver regeneration (8).

Although primary embryonic neural cells can spontaneously cluster and migrate along axons and radial glia fibers (12) they do not grow in multiple layers. On the contrary when multicellular cell clusters are put on a cultivation dish, the neural cells prefer to emigrate and spread in a monolayer. While nervous cells are postmitotic, neural progenitor and stem cells represent an ideal source that can generate any neural cells. The neurosphere assay established by Reynolds and Weiss (23) was used to maintain the cells in the undifferentiated phenotype in case the neurospheres are passaged regularly. Cell differentiation is prevented by regular cell dissociation while the resulting cells are not allowed to anchor to a substrate. Mitogenic effects of epidermal and fibroblast growth factors drive proliferation of progenitor 
and stem cells. When grown in small cell densities, each neurosphere arises as a cell clone. The size of neurosphere derived from neural progenitor is different from those derived from stem cells (24). Simultaneous growth factor stimulation leads to symmetric divisions of undifferentiated cells inside of neurospheres and to cell expansion. In the growing neurosphere, the resulting cell number is balanced by cell proliferation and cell death. The cells in the neurosphere grown without passaging undergo spontaneous cell differentiation that reflects characteristic zonal arrangement: immature cells incl. progenitors occupy the peripheral belt whereas neurons and astroglia are localized in the central core. Cells of the long-term neurospheres can reach their terminal differentiation. Oligodendrocytes are able to cover the axons with myelin sheaths and neurons make interneuronal synapses containing synaptic vesicles. Astrocytes can form perivascular limiting membrane as demonstrated by intracerebral transplantation of solid neurospheres $(14,15)$. Similar microscopic structure and basic cell arrangement can be expected in other spheroids grown in vitro including cardiospheres and mammospheres $(1,2)$.

Another strategy how to prepare 3D culture from the cells that do not stratify or form spheroids is to provide them a support in a form of 3D latticework using suitable gels or scaffolds. Matrigel consists of a mixture of gelatinous glycoproteins (e.g., laminin, collagen, entactin etc.) enriched for bioactive molecules (e.g., growh factors, metalloproteinases) isolated from mouse sarcoma. In cell cultures Matrigel was used to navigate the endothelial cells to form $3 \mathrm{D}$ tubes mimicking the blood vessels (21) or to grow the primitive alveoli by epithelial cells of the mammary gland (17). A good example of artificial gels is a hydrogel based upon polyethyleneglycol and fibrinogen that promotes muscle fibre formation after short-term cultivation with mesoangioblasts (7).

Biogenic scaffolds are superior to fabricated artificial materials. Tissue decellularization (9) offers an alternative to obtain scaffolds formed by the extracellular matrix that reflect tissue microstructure and contain bioactive molecules supporting cell attachment and organisation. Moreover, acellular tissue scaffoldings offer several potential advantages as they are immunologically inert, retain extracellular proteins, basal laminas, and stromal components that facilitate cell incorporation in vivo. Moreover, they contain remnants of the acellular native vascular network that allow to perfuse the construct. Our short-term in vitro study of cell colonization of scaffolds obtained from a decellularized skeletal muscle demonstrates their good biocompatibility. C2C12 cells that adhere and migrate over the surfaces eventually enter the scaffold interior to be organized in three dimensions. The immigrating cells are not arranged randomly; instead they are organized along the inner parallel tube-like spaces. The cell density of recellularized scaffolds does not correspond to density of neurospheres or tightly stratified layers of WRL 68 cells grown in PetriPerm dishes but this obstacle could be solved by pretreatment with chemoattractant and growth factors or by a careful selection of cells used for recellularization. On the other hand, the cells inside the muscle scaffolds remain viable, do not show any abnormal changes, any signs of regression or cell death, which speaks in favour of biogenic scaffolds derived from the tissue extracellular matrix. Recently several groups established a proof of concept for utilization of decellularized bioscaffold reseeded with cells in reengineering of complex bioartificial organs like the heart (19), lung (20) or liver (25). Such organs are transplantable and functional as demonstrated by Uygun et al. 2010 in liver grafts (25) or by Olausson et al. 2012 in veins (18).

\section{Conslusion}

There exist many more alternatives to methods that permit the researchers to grow the cells in 3D culture systems, especially the cells with a low proliferative and migration capacity. Some are based on forced or passive cell aggregation examples of which include culturing the cells in rotating bioreactors or hanging drops used to produce embryoid bodies. Rotating bioreactors facilitating diffusion of cultivation medium are useful in short-term organotypic cultures $(4,13)$. Cell 3D printing became a novel sophisticated tool that allows precise positioning of cells in space in combination with selection of suitable biomolecules to create optimal microenvironment that mimics microarchitecture of native tissues (16). Combination of 3D culturing with a promising potential of pluripotent stem cells opens a new avenue for generation of organoids or miniature organs. Recent reports announced a successful construction of several mini-organs including renal, intestinal and cerebral organoids $(11,27,28)$.

$3 \mathrm{D}$ cell constructs generated in vitro mimic embryonic development, closely resemble tissue morphology and also display excellent functional characteristics, which determine these powerful systems to model organogenesis, disease pathogenesis, screen drugs, test new therapies and even to construct replacement tissues.

\section{Acknowledgements}

This work was supported by the European Social Fund, the state budget of the Czech Republic Project No. CZ.1.07/2.3.00/30.0061, Prvouk P37/06 and SVV-2015260179 .

\section{References}

1. Beltrami AP et al. Adult cardiac stem cells are multipotent and support myocardium regeneration. Cell 2003; 114: 763-76.

2. Cioce $\mathrm{M}$ et al. Mammosphere-forming cells from breast cancer cell lines as a tool for the identification of CSC-like- and early progenitor-targeting drugs, Cell Cycle 2010; 9: 2950-9.

3. Cizkova D, Mokry J, Micuda S, Osterreicher J, Martinkova J. Expression of MRP2 and MDR1 transporters and other hepatic markers in rat and human liver and in WRL 68 cell line. Physiol Res 2005; 54: 419-28. 
4. Freed LE, Vunjak-Novakovic G. Tissue culture bioreactors: Chondrogenesis as a model system. In: Lanza RP, Langer R, Chick WL eds. Principles of tissue engineering. Austin, USA: Academic Press, 1997: 151-65.

5. Freshney RI. Culture of specific cell types. In: Culture of animal cells: A manual of basic technique (2nd ed.). New York: Alan R. Liss Inc., 1988: 257-80.

6. Freshney RI. Three-dimensional culture systems. In: Culture of animal cells: A manual of basic technique (2nd ed.). New York: Alan R. Liss Inc., 1988: 297-307.

7. Fuoco $\mathrm{C}$ et al. In vivo generation of a mature and functional artificial skeletal muscle. EMBO Mol Med 2015; 7: 411-22.

8. Hoehme $\mathrm{S}$ et al. Prediction and validation of cell alignment along microvessels as order principle to restore tissue architecture in liver regeneration. Proc Natl Acad Sci USA 2010; 107: 10371-6.

9. Hrebikova H, Diaz D, Mokry J. Chemical decellularization: A promising approach for preparation of extracellular matrix. Biomed Pap 2015; 159: 12-7.

10. Laerum OD, Bjerkvig R. Monolayer and three-dimensional culture of rat and human central nervous system: Normal and malignant cells and their interactions. Meth Neurosci 1990; 2: 210-36.

11. Lancaster MA, Knoblich JA. Generation of cerebral organoids from human pluripotent stem cells. Nat Protoc 2014; 9: 2329-40.

12. Liour SS, Yu RK. Differentiation of radial glia-like cells from embryonic stem cells. Glia 2003; 42: 109-17.

13. Mazzoleni G, Di Lorenzo D, Steimberg N. Modelling tissues in 3D: The next future of pharmaco-toxicology and food research? Genes Nutrition 2009; 4: 13-22.

14. Mokry J, Karbanova J, Filip S. Differentiation potential of murine neural stem cells in vitro and after transplantation. Transplant Proc 2005; 37: 268-72.

15. Mokry J, Subrtova D, Nemecek S. Differentiation of epidermal growth factor-responsive neural precursor cells within neurospheres. Acta Med 1996; 39: 7-20.
16. Murphy SV, Atala A. 3D bioprinting of tissues and organs. Nature Biotechnol 2014; 32: 773-85.

17. Murtagh J, McArdle E, Gilligan E, Thornton L, Furlong F, Martin F. Organization of mammary epithelial cells into 3D acinar structures requires glucocorticoid and JNK signaling. J Cell Biol 2004; 166: 133-43.

18. Olausson $\mathrm{M}$ et al. Transplantation of an allogeneic vein bioengineered with autologous stem cells: A proof-of-concept study. Methods Mol Biol 2012; 798: 21-52.

19. Ott HC et al. Perfusion-decellularized matrix: Using nature's platform to engineer a bioartificial heart. Nat Med 2008; 14: 213-21.

20. Petersen TH et al. Tissue-engineered lungs for in vivo implantation. Science 2010; 329: 538-41.

21. Ponce ML. Tube formation: an in vitro matrigel angiogenesis assay. Methods Mol Biol 2009; 467: 183-8.

22. Qing Q, Qin T. Optimal method for rat skeletal muscle decellularization. Chin J Rep Rec Surg 2009; 23: 836-9.

23. Reynolds BA, Weiss S. Generation of neurons and astrocytes from isolated cells of the adult mammalian central nervous system. Science 1992; 255: 1707-10.

24. Reynolds B, Rietze RL. Neural stem cells and neurospheres - re-evaluating the relationship. Nature Methods 2005; 2: 333-6.

25. Uygun BE et al. Organ reengineering through development of a transplantable recellularized liver graft using decellularized liver matrix. Nat Med 2010; 16: 814-20.

26. von der Mark K, Gauss V, von der Mark H, Müller P. Relationship between cell shape and type of collagen synthesised as chondrocytes lose their cartilage phenotype in culture. Nature 1977; 267: 531-2.

27. Watson CL et al. An in vivo model of human small intestine using pluripotent stem cells. Nature Medicine 2014; 20: 1310-4.

28. Xia Y et al. Directed differentiation of human pluripotent cells to ureteric bud kidney progenitor-like cells. Nat Cell Biol 2013; 15: 1507-15.

Received: 16/06/2015 Accepted in revised form: 09/09/2015

\section{Corresponding author:}

Prof. MUDr. Jaroslav Mokrý, Ph.D., Charles University in Prague, Faculty of Medicine in Hradec Králové, Šimkova 870, 50038 Hradec Králové, Czech Republic; e-mail: mokry@lfhk.cuni.cz 